\title{
La contribution potentielle des énergies renouvelables au développement durable : le cas de l'hydroélectricité
}

- Renewable energy technologies potential contribution to sustainable development : the example of hydro power -

par Bernard Chabot, Expert Energies renouvelables

ADEME

\section{L'hydroélectricité constitue la première filière de production d'électricité primaire à l'échelle mondiale et compte tenu du poten- tiel exploitable, devrait contribuer au développement durable.}

Un recours plus important aux énergies renouvelables est une des conditions nécessaires pour prendre le chemin d'un développement durable, du fait de leurs avantages pour résoudre les problèmes d'énergie, d'environnement et de développement économique et social [1].

Les énergies renouvelables sont basées sur l'exploitation de flux naturels d'énergie : rayonnement solaire, cycle de l'eau, des vents et du carbone dans la biosphère, flux de chaleur interne de la terre, effet de l'attraction lunaire et solaire sur les océans. Ce sont donc des éner- gies inépuisables, à l'inverse des énergies fossiles et minières (charbon, pétrole, gaz naturel, uranium) dont l'accès aux réserves à faibles coûts, par nature limitées et souvent mal réparties, accentue les tensions géopolitiques présentes et surtout à venir.

Elles n'émettent pas de gaz à effet de serre (y compris dans le cas d'une exploitation rationnelle de la biomasse, où le rapport émission/fixation de carbone peut être quasiment équilibré). Leur substitution aux énergies fossiles carbonées réduit donc le risque de réchauffement climatique. Leur impact sur l'environnement local est par ailleurs limité, jamais irréversible et toujours limité à la période d'exploitation du fait de l'absence de problèmes particuliers de démantèlement et de gestion de déchets toxiques et de longue durée de vie. Enfin les précautions, les règles de l'art et les solutions techniques qui permettent de limiter leur impact sur l'environnement local sont bien connues et facilement intégrables dans les règlements et procédures d'autorisation des projets.

Renewable energy technologies offer many advantages for energy supply and sustainable development. Meanwhile, their large scale deployment is often difficult to achieve due to the harsh competition with fossil fuels, both for comfort and ease of use for decision makers and users and for direct cost competitiveness, which often do not take into account the social and environmental costs. So, the example of Hydro Power which is still the main non fossil source for electricity in the world and which continues to develop strong markets is presented here as a success story which could be repeated in the short and medium term by other renewable energy technologies. Moreover, as only a small part of the world hydro technical potential has been tapped, the hydropower contribution to sustainable development will be secured in the future, as well as for large scale projects including new technologies and behaviour to lower potential impacts on local environment and for small hydropower projects well designed for rural electrification in developing countries.

Cette communication est reproduite avec l'aimable autorisation du Centre universitaire d'étude des problèmes de l'énergie de l'Université de Genève (CUEPE). Elle est limitée ici au cas de l'hydroélectricité, et le lecteur intéressé aussi par le cas de l'éolien et de l'électricité photovoltaïque pourra se reporter utilement à sa version intégrale figurant dans les actes de la journée du CUEPE 1997 "Quelle place pour les énergies renouvelables au siècle prochain", Genève, 30 octobre 1997. 
Les énergies renouvelables permettent de produire une grande diversité de produits et de vecteurs énergétiques : énergie thermique, combustibles solides, liquides et gazeux, électricité. Cette diversité d'applications leur permet de pénétrer le marché des produits et des services énergétiques de tous les secteurs économiques : agriculture, industrie, habitat et tertiaire, transports.

Cette diversité d'applications ainsi que la complémentarité des gisements (soleil, vent, eau, biomasse...) et leur bonne répartition de par le monde permet une utilisation décentralisée des énergies renouvelables, à la fois spatialement et au niveau des décideurs et des utilisateurs : familles, villages, cités, entreprises, régions et pays, que ces derniers soient industrialisés ou en développement. La valorisation de cette production décentralisée peut se faire par le biais de réseaux existants : réseaux d'électricité, de gaz, de chaleur, de transport et de commercialisation de combustibles. Mais l'utilisation décentralisée des énergies renouvelables permet aussi et surtout d'apporter le minimum de services énergétiques modernes dans les régions ou les pays en développement où les réseaux conventionnels de distribution d'énergie ne pénétreront pas avant des décennies.

Les techniques requises pour exploiter les énergies renouvelables vont des techniques traditionnelles (le bois de feu), à des techniques maîtrisables par tous les pays où des industries électromécaniques et chimiques sont déjà établies (hydroélectricité, énergie éolienne, capteurs solaires, biocombustibles et biocarburants...) et enfin à des techniques de pointe mais qui ont déjà donné lieu à des transferts de technologies réussis entre pays industrialisés et pays en développement, comme dans le cas de la fabrication locale de cellules et de modules photovoltaïques. Les transferts et l'appropriation des technologies d'énergies renouvelables sont donc possibles entre les pays industrialisés et les pays émergents ou en développement, sans compter les possibilités de coopération "Sud/Sud".

Malgré le fait qu'elles soient basées sur des ressources inépuisables et souvent "gratuites" (soleil, vent...), les énergies renouvelables nécessitent pour être exploitées des ressources en capital, en matières premières et en espace. Dans une optique d'utilisation à grande échelle, leur exploitation rationnelle doit donc se concevoir comme le couronnement d'une démarche globale de mầtrise de l'énergie basée sur les principes suivants :

* La Sobriété énergétique, qui permet de déterminer quels sont les services énergétiques indispensables et de leur affecter prioritairement les ressources disponibles par rapport à ceux qui sont superflus.

* L'efficacité énergétique, qui permet d'assurer ces services énergétiques prioritaires en consommant le moins possible d'énergie primaire (d'origine fossile ou renouvelable). Les solutions techniques correspondantes sont maintenant bien connues, tant du côté de la production d'énergie (par exemple la cogénération) que du côté de sa transformation et de son utilisation (par exemple les lampes à fluorescence qui consomment cinq fois moins d'énergie que les lampes à incandescence). La synergie entre la sobriété énergétique et l'efficacité énergétique débouche sur ce que l'on peut appeler à juste titre l'utilisation rationnelle de l'énergie.

* Le respect de l'environnement, par l'utilisation de ressources énergétiques qui font peser le moins possible de contraintes sur l'environnement local, régional et planétaire et sur les générations futures, soit en épuisant d'ici moins d'un siècle des stocks de ressources naturelles non renouvelables, soit en nécessitant une mobilisation continue pour gérer des déchets à longue durée de vie.

Les énergies renouvelables exploitées rationnellement et équitablement, répondent en particulier à ces principes et à ces critères.

Compte tenu de ces avantages et des progrès techniques et économiques amenés par les programmes de recherche et développement et d'ouverture des marchés consentis depuis les premiers chocs pétroliers, on peut se demander pourquoi la contribution des énergies renouvelables reste inférieure à $20 \%$ des besoins totaux d'énergie primaire mondiaux, soit environ $6 \%$ via l'hydroélectricité (2 $300 \mathrm{TWh} / \mathrm{an}$, soit $510 \mathrm{Mtep}$ ) et $13 \%$ via la biomasse (1,3 Gtep dont 1,1 Gtep dans les pays en développement, principalement sous forme de "bois de feu"), les autres filières (solaire thermique et photovoltaïque, énergie éolienne, géothermie...) comptant pour encore moins de $1 \%$ malgré des taux de croissance élevés. A cela il y a plusieurs raisons.

La concurrence et la compétitivité des énergies fossiles s'est accrue depuis le contre-choc pétrolier en 1986. De plus, les filières correspondantes ont bénéficié de progrès importants. Par exemple la mise au point et la commercialisation de centrales électriques au gaz naturel utilisant des cycles combinés à haut rendement (plus de $50 \%$ ) et de faible coût d'investissement (moins de 4 FRF/W) provoque actuellement une "ruée vers le gaz" de nombreuses compagnies d'électricité de par le monde, au détriment de technologies classiques n'émettant pas de gaz à effet de serre mais plus gourmandes en investissement initial comme l'hydroélectricité et le nucléaire.

Changer le "paysage énergétique mondial" requiert des générations : il a fallu 100 ans d'investissements massifs à l'hydroélectricité pour dépasser $2300 \mathrm{TWh}$ par an ; après cinquante ans d'investissements encore plus massifs, la contribution mondiale de l'énergie nucléaire (2200 TWh/an) est limitée à moins de $6 \%$ des besoins totaux d'énergie primaire. Mais c'est justement une raison de plus de ne pas remettre aux lendemains les efforts de diversification vers des sources d'énergie n'émettant pas de gaz à effet de serre !

Les prix de l'énergie sont généralement basés sur des coûts comptables qui prennent en compte les dépenses d'investissement, d'exploitation et d'entretien-maintenance, mais qui ne prennent pas en compte ce que l'on appelle les "externalités". Ces dernières recouvrent les coûts pour l'environnement local régional et planétaire et les coûts pour la santé et la société induits par la production et la consommation de l'énergie. On comprend aisément que ces externalités peuvent ne pas être négligeables et que leur prise en 
compte puisse orienter différemment les choix des producteurs et des consommateurs d'énergie. Mais il ne faut pas attendre de "miracle" des calculs de ces externalités : leurs valeurs ne peuvent être approchées qu'à la suite d'hypothèses qualitatives (par exemple la valeur donnée à un biotope ou à une vie humaine) et attendre qu'on aboutisse à un consensus sur les externalités dans chaque configuration technique et d'implantation reviendrait à décider de ne rien faire pendant des années. Plus que leur niveau absolu, c'est plutôt le "différentiel d'externalités" entre les énergies renouvelables et les énergies fossiles qui est intéressant à analyser. Comme on le voit en figure 1, il est très en faveur des énergies renouvelables, y compris pour la biomasse valorisée en cogénération. Ainsi, à chaque fois que des taxes ou des incitations ont été mises en place pour donner un signal clair sur l'existence de ce différentiel d'externalités, le résultat a été largement positif pour les énergies renouvelables, comme on a pu le voir en Europe dans le cas de l'énergie éolienne.

La compétitivité économique est nécessaire mais pas suffisante pour qu'une filière énergétique puisse se développer à grande échelle. Des conditions techniques, réglementaires, législatives et tarifaires adaptées sont aussi indispensables. En particulier, des conditions simples et de faible coût doivent permettre l'accès aux "réseaux" au sens large des filières d'énergies renouvelables décentralisées de petite et moyenne puissances (petites centrales hydroélectriques, aérogénérateurs, toits photovoltaïques...).

Enfin, du fait de l'inertie des marchés de l'énergie et de la position prépondérante des énergies fossiles d'une part et de l'aspect décentralisé des applications les plus courantes des énergies renouvelables, le développement à grande échelle de ces dernières requiert un investissement préalable important pour l'information, la motivation et la formation des millions ou des milliards d'usagers potentiels. Ainsi, la majorité des foyers ruraux des pays en développement utilisant du bois de feu, des lampes à pétrole et des piles jetables verraient leur conditions de vie largement améliorées, souvent sans dépenses cumulées d'investissement et de fonctionnement supplémentaires, si elles s'équipaient en "foyers améliorés" (permettant de diviser par deux ou trois la consommation annuelle de bois) et en petits systèmes photovoltaïques domestiques pour l'éclairage et la radio. Or ces investissements préalables pour l'information et la formation des usagers et des relais techniques et commerciaux locaux ne peuvent pas la plupart du temps être pris en charge par le secteur privé, soit parce que la valeur du produit est trop faible (foyers améliorés artisanaux) soit parce que le marché est encore trop limité pour pouvoir amortir les frais correspondants sur des petites séries (cas des "kits photovoltaïques"). De leur côté, les pouvoirs publics ont à faire face avec des moyens limités au problème global du développement économique de ces zones (agriculture, désenclavement, santé, éducation...) et ils ne peuvent pas toujours accorder toute l'attention nécessaire aux problèmes énergétiques, bien qu'on retrouve une composante "énergie" dans tous les volets du développement cités ci-dessus.

Dans les pays industrialisés, ce problème n'est pas non plus négligeable : alors qu'il est relativement aisé à un fournisseur de centrales à cycles combinés de convaincre quelques grandes compagnies d'électricité des avancées et de la fiabilité de sa nouvelle technologie, convaincre un

\section{Externalités de la production d'électricité en Europe.}

CC : Cycles combinés :

ST : $\quad$ turbine à vapeur :

GT : $\quad$ turbine à gaz :

BIG/ISTIG : biomasse gazéifiée, injection vapeur dans GT.

Nota l : basé sur le coût d'une vie humaine de $17 \mathrm{MFRF}$, appliqué sans prorata du nombre d'années perdues.

Nota 2 : centrales conformes aux règlementations actuelles.

Nota 3 : incertitudes élevées, notamment sur valeurs minimales.

(Source SI : étude DG XII ExterE, rapport préliminaire, 1995. Autres : "Extension de l'analyse Impact Pathways aux coûts externes des installations de cogénération", ARMINES, 10/96).

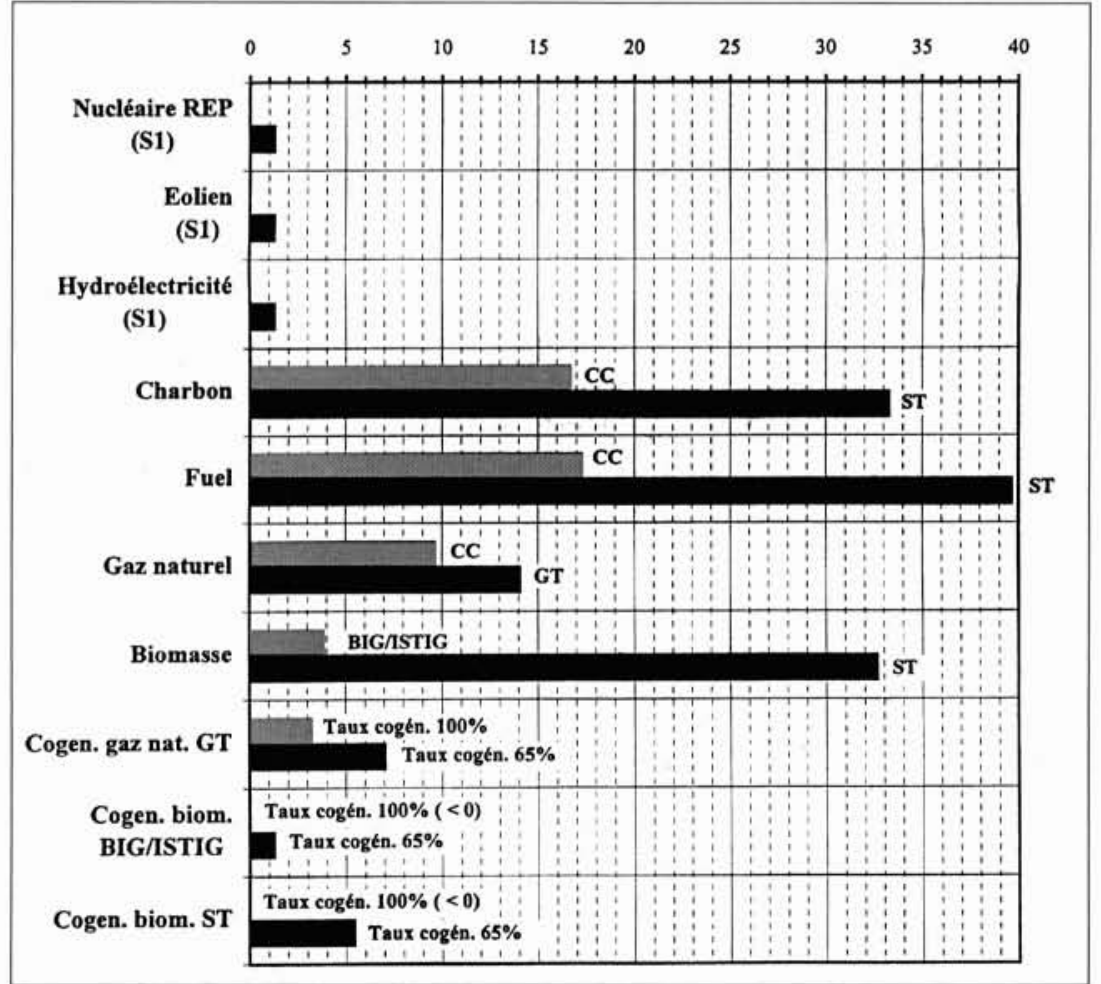


pourcentage suffisant de ménages ou de gestionnaires de bâtiments de l'intérêt des chauffe-eaux solaires individuels ou collectifs nécessite un investissement commercial souvent hors de portée de petites et moyennes entreprises du secteur, qui ne pouvant ainsi augmenter leurs séries de production ne peuvent diminuer leurs coûts de façon suffisante.

Tout se passe ainsi comme si l'on était face à un "cercle vicieux" : le marché accessible est étroit et insuffisant pour permettre des baisses de coûts par effet de série, et les prix restant élevés, le marché ne décolle pas !

Heureusement, les énergies renouvelables ne sont pas condamnées à cette logique implacable, et on va voir que des solutions existent pour briser ces cercles vicieux et pour "passer à des cercles vertueux", avec pour ces derniers une rétroaction très fortement positive entre l'ouverture des marchés et les baisses de coûts, amplifiée par la possibilité de consacrer plus de fonds propres à la recherche, au développement de nouveaux produits et services et à la promotion commerciale.

Pour cela, rien ne vaut des exemples de réussites, qui seront pris ici dans le secteur de l'électricité, domaine stratégique puisque concernant la forme la plus noble de l'énergie (pouvant donner accès notamment à l'éclairage, à la force motrice et surtout au traitement et à la transmission de l'information pour l'éducation, la formation, la culture, les loisirs et les activités professionnelles) et faisant partie d'un des secteurs de l'énergie en forte croissance et participant fortement aux émissions de gaz à effet de serre.

Un exemple de réussite : l'hydroélectricité à grande et à petite échelle

Il est souvent de bon ton de faire une différence marquée entre les applications à moyenne et grande puissance de l'hydroélectricité (de quelques dizaines de MW à plusieurs $\mathrm{GW}$ ) et la petite hydroélectricité (moins de 5 ou moins de $25 \mathrm{MW}$ selon les pays). A l'extrême, seule cette dernière est parfois jugée digne de recevoir la qualification d'énergie renouvelable et de figurer dans les statistiques et les programmes ad-hoc ! Cette position est bien sûr absurde, et souvent pas innocente, puisqu'elle permet de "démontrer" que les énergies renouvelables ainsi artificiellement réduites ne fournissent qu'un pourcentage négligeable de l'électricité primaire mondiale, alors que justement, avec l'hydroélectricité elles représentent la première source d'électricité primaire dans le monde. Bien sûr, il n'est pas question de passer sous silence les quelques contre références de grands projets d'hydroélectricité réalisés en leur temps sans apporter une attention suffisante aux problèmes sociaux et d'environnement, mais plutôt de rappeler quelques évidences qui peuvent éclairer et orienter le développement de cette filière au XXIe siècle.

Comme on le voit en figure 2 , le développement de I'hydroélectricité est toujours soutenu, et ses perspectives d'utilisation mondiales ne sont pas bornées par le potentiel technique et économique, dont seulement $1 / 7^{\text {ème }}$ est actuellement exploité. Et surtout, de grands pays consommateurs d'énergie au XXIe siècle (Chine, Indonésie, Brésil, Zaïre...) ont des potentiels énormes et à peine entamés.

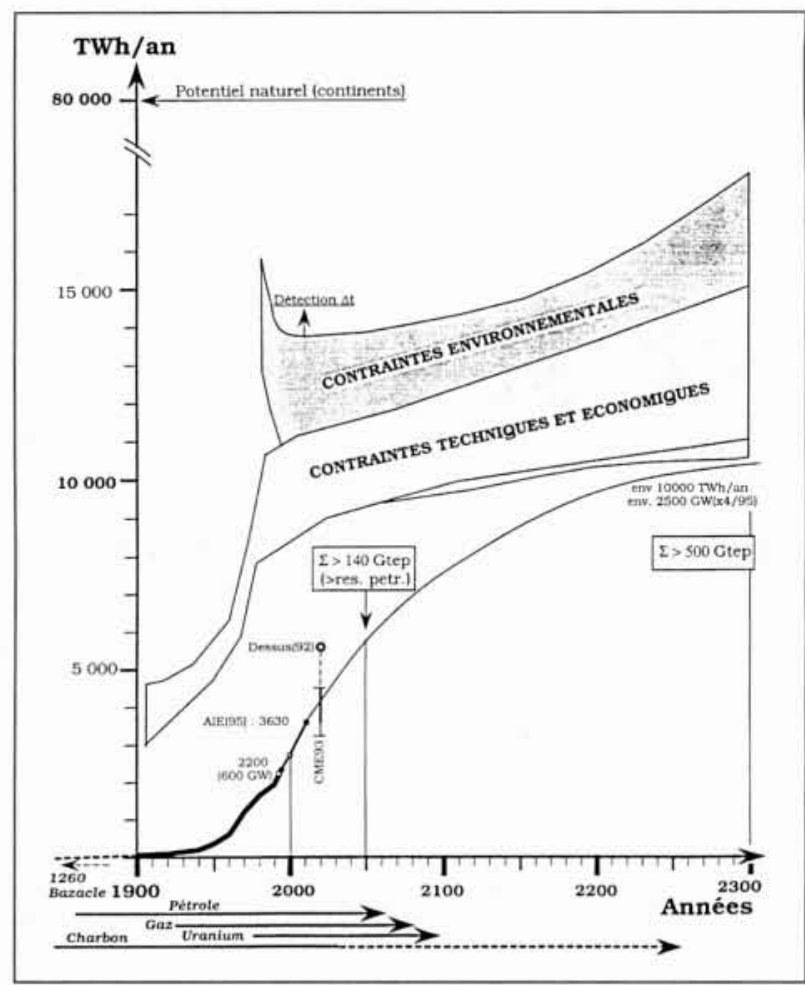

2. L'hydroélectricité : historique, pistes pour le futur. En 2050, la production cumulée probable d'hydroélectricité sera équivalente aux réserves prouvées actuelles de pétrole.

La démonstration a été faite, notamment par la France et par la Suisse, qu'un pays peut exploiter plus de $90 \%$ de son potentiel hydroélectrique sans problèmes majeurs d'environnement, et au contraire en valorisant et en enrichissant son patrimoine et en permettant une utilisation plus rationnelle de l'eau pour d'autres usages : réduction des crues, irrigation, adductions d'eau, loisirs. Ainsi en France, le volume d'eau stockée dans les retenues des aménagements hydroélectriques dépasse 7,5 milliards de $\mathrm{m}^{3}$, soit les trois quarts des réserves d’eau constituées en surface sur le territoire national.

L'évolution des techniques (par exemple les groupes turboalternateurs immergés pour très faibles chutes) permettent des "aménagements doux" des fleuves, en évitant les grands barrages par des dérivations (cas de l'aménagement du Rhône en aval de Lyon), voire sans aucune dérivation et par simple aménagement des rives (centrale sur l'Isère en aval de Grenoble, projets d'aménagement du Rhône en amont du lac Léman).

Les progrès de l'interconnexion des grands réseaux électriques, du transport de l'électricité à grande distance ou de la fabrication, du transport et de l'utilisation de nouveaux vecteurs énergétiques (hydrogène, méthanol...) permettront l'exploitation de "mégagisements" hydroélectriques à un coût du $\mathrm{kWh}$ extrêmement faible. Enfin, de nouvelles approches permettent de réduire et de mieux traiter les problèmes posés par le déplacement de populations lors de projets hydroélectriques avec réservoirs [2]. 
Cette nécessaire remise en perspective du développement global de l'hydroélectricité ne doit bien sûr pas mener à négliger les efforts à consacrer au développement de la petite hydroélectricité (moins de $10 \mathrm{MW}$ ). Celle-ci présente en effet des avantages spécifiques, notamment de pouvoir donner lieu à des investissements de plus petite taille, donc plus accessibles aux collectivités locales et présentant des impacts plus faibles sur l'environnement. Un recours accru à cette filière, en particulier dans les pays en développement, présente un intérêt stratégique compte tenu du rôle que les $\mathrm{PCH}$ (petites centrales hydroélectriques) peuvent jouer pour l'électrification rurale décentralisée [3]. L'exemple de la Chine est à cet égard tout à fait intéressant à analyser [4].

De 1974 à 1993, le développement de la petite hydroélectricité en Chine a été spectaculaire : la puissance installée est passée de 2,6 à $15 \mathrm{GW}$ (une multiplication par 5,7 ) et l'énergie produite est passée de 4,87 à $47 \mathrm{TWh}$ par an (une multiplication par pratiquement un facteur 10 , traduisant l'amélioration technique des équipements et de l'exploitation). Sur les 48284 petites centrales hydroélectriques répertoriées en 1993, plus de $85 \%$ sont gérées au niveau des villages. Moins de $8 \%$ du total sont reliées au réseau national interconnecté et les autres alimentent des réseaux locaux $(30 \%)$, ou fonctionnent de façon totalement autonome pour électrifier un seul village (30 000 soit $62 \%$ ). Cette électrification ne se limite pas à la production d'électricité, mais elle s'est accompagnée d'actions sur l'utilisation sobre et efficace de l'énergie :

— priorité accordée aux besoins productifs : agriculture (irrigation, moulins...), petites industries et artisanat, de façon à faciliter le "décollage économique" par l'électrification rurale,
- promotion d'appareils domestiques efficaces, notamment de cuiseurs à riz de faible puissance pour limiter le recours au bois de feu lorsque l'eau est abondante et pour éviter la création de pointes de consommations et mieux valoriser la production en heures creuses.

Par ailleurs, la gestion des programmes correspondants a été largement décentralisée, aussi bien au niveau technique (ateliers de production des équipements, études et réalisations des projets) qu'à celui de la gestion (financements de l'investissement initial, exploitation, tarification et vente du courant, réinvestissement des bénéfices pour d'autres opérations de petite hydroélectricité). De plus, depuis le début des années 90 , les promoteurs et les propriétaires de petites centrales hydroélectriques peuvent être aussi bien des collectivités locales que des coopératives ou des investisseurs privés et tous sont libres de vendre l'électricité au tarif qui leur convient le mieux.

Cette approche décentralisée a ainsi permis d'électrifier plus de 90 millions de personnes depuis 1980. Bien que la décentralisation ait permis de rendre marginale la contribution de l'Etat pour le financement des investissements et de rendre inutile le recours à des financements internationaux, le rôle des pouvoirs publics a été et reste crucial pour fixer les "règles du jeu" et pour prendre en charge l'énorme effort de formation et d'information qui a rendu possible ce succès. Juste retour des choses, le "point focal" de ces actions, le "Hangzhou Regional Center (HRC)" est maintenant reconnu au niveau international et par les Nations-Unies comme centre de référence pour la promotion de la petite hydroélectricité.

Comme la contribution mondiale de la petite hydroélectricité est de l'ordre de 80 TWh par an alors que le gisement mondial exploitable est de l'ordre d'au moins

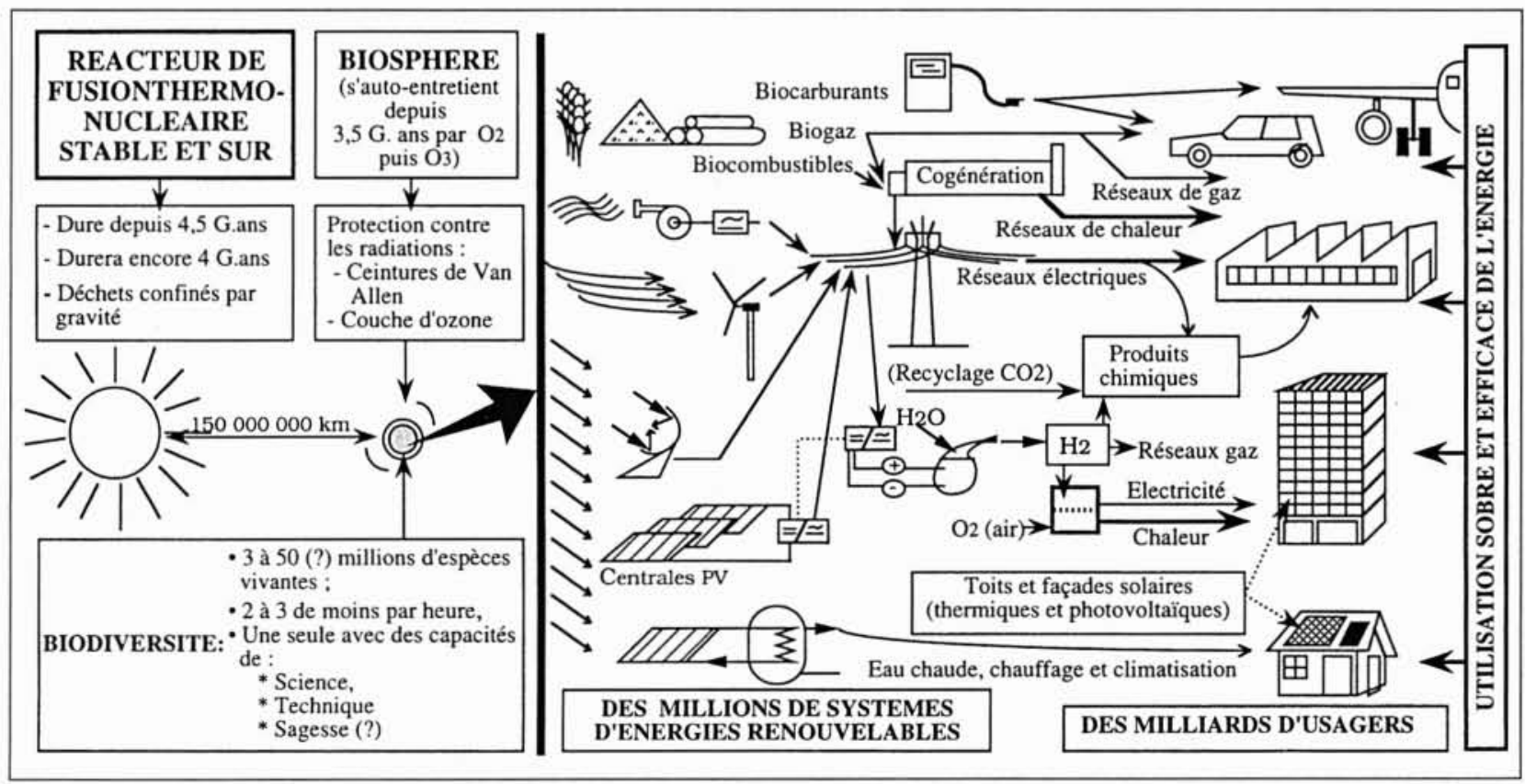

3. L'intégration à long terme et à grande échelle des énergies renouvelables : vers la "fusion bien tempérée". 
$500 \mathrm{TWh}$ par an, on voit que le potentiel de contribution de cette forme d'énergie renouvelable à l'électrification et au développement économique et social des zones rurales des pays en développement en est seulement à ses débuts et que d'autres "cercles vertueux" de ce type pourraient et devraient être créés dans d'autres pays.

Ces exemples de créations réussies ou en cours de "cercles vertueux" ne sont pas limitatifs. En particulier, on assiste actuellement à un développement très rapide et soutenu de l'énergie éolienne et de l'électricité photovoltaïque. L'extension de ces réussites aux autres applications des énergies renouvelables proches de la rentabilité économique ou correspondant à une demande sociale et à d'autres secteurs économiques des pays industrialisés et en développement ne poserait pas de problèmes insurmontables, mais au contraire amènerait sur le moyen et le long terme des bénéfices sociaux, économiques et environnementaux certains.

Cette généralisation passe par l'intégration des énergies renouvelables dans la démarche globale de maîtrise de l'énergie décrite dans l'introduction et dans les politiques environnementales et de coopération entre les pays industrialisés et les pays en développement. La dynamique ainsi créée pourra de plus se renforcer continûment des progrès apportés par la recherche et le développement.

Ainsi sur le moyen et le long terme, l'intégration de plus en plus poussée des applications des énergies renou- velables dans tous les secteurs économiques et en synergie avec l'utilisation sobre et efficace de l'énergie mèneront à ce que l'on peut d'ores et déjà appeler la "fusion bien tempérée" (cf. figure 3 ) : s'il n'y a aucun doute que sur le très long terme, l'humanité devra appuyer son développement sur l'énergie issue de la fusion thermonucléaire, le recours aux applications directes et indirectes de l'énergie du soleil - qui est lui-même un réacteur thermonucléaire dont l'énergie est accessible partout, de façon sûre et de plus en plus économique - est une solution évidente et rationnelle si l'on veut vraiment prendre le chemin du développement durable.

\section{RÉFÉRENCES:}

[1] B. Chabot, "Les énergies renouvelables", CD-ROM "Encyclopaedia Universalis", Version 3, Paris, 1997.

[2] Michael M. Cernea. "Finding Sound Approaches to Hydro Project Resettlement", Hydro Review Worldwide, Vol. 5, N 6, 1997, Kansas City. USA.

[3] Bernard Chabot: "L'électrification rurale décentralisée : état de l'art et perspectives techniques et économiques". Revue de l'Energie, $N^{\circ} 475$. Paris, 1996.

[4] Tong Jiandong: "Small Hydropower: China's Practice". Hangzou Regional Center Publications, 1994 